\title{
ACCRA Project: Agile Co-Creation for Robots and Aging.
}

\author{
Laura Fiorini ${ }^{1{ }^{*},}$, Grazia D’Onofrio ${ }^{1,2}$, Raffaele Limosani ${ }^{1}$, Daniele Sancarlo ${ }^{2}$, \\ Antonio Greco ${ }^{2}$, Francesco Giuliani ${ }^{3}$, Antonio Kung ${ }^{4}$, Paolo Dario ${ }^{1}$, Filippo \\ Cavallo $^{1}$
}

\begin{abstract}
The mission of ACCRA (Agile Co-Creation for Robots and Aging) is to enable the development of advanced ICT Robotics based solutions for extending active and healthy aging in daily life by defining, developing and demonstrating an agile co-creation development process. ACCRA project consists of three robotic applications which aim to promote the independent living by means of personal mobility application, to support the daily life management thanks to housework application and to promote conversation rehabilitation tailored on personal attitude by means of dedicated software programme. Additionally, ACCRA project will be designed and developed on open source framework (i.e. ROS, FIWARE, universAAL and Rospex) to promote the interoperability among scientific community.
\end{abstract}

Keywords: Service Robotics, Agile Programming, Co-Creation, Active Aging

\section{Introduction}

European population is getting older. This is the result of a simultaneous drop in fertility rates, longer life expectancies and a shift of the post-war baby boom generations to the top of the age pyramid. In particular, by 2060, the demographic old-age dependency ratio is projected to rise from the current $28 \%$ to $50 \%$ [1].

\footnotetext{
${ }^{1}$ The BioRobotics Institute, Scuola Superiore Sant'Anna, Pontedera (PI), Italy; e-mails: \{raffaele.limosani, paolo.dario, filippo.cavallo\}@santannapisa.it.

${ }^{2}$ Complex Unit of Geriatrics, Department of Medical Sciences, IRCCS "Casa Sollievo della Sofferenza", San Giovanni Rotondo, Foggia, Italy; e-mails \{g.donofrio, d.sancarlo, a.greco\}@operapadrepio.it.

${ }^{3}$ ICT, Innovation and Research Unit, IRCCS "Casa Sollievo della Sofferenza", San Giovanni Rotondo, Foggia, Italy; e-mail: f.giuliani@operapadrepio.it.

${ }^{4}$ Trialog, Paris, France; e-mail: antonio.kung@trialog.com.

*corresponding author: Laura Fiorini e-mail: laura.fiorini@santannapisa.it.
} 
Consequently, this demographic shift implies the understanding of healthy aging and age-related diseases as one of our future challenges.

"European innovation partnership on active and healthy aging action group" states that older people can age in better physical and mental health within agefriendly environments [2]. These environments would promote the social inclusion of elderly population. In this context, robots can contribute to make those environments more friendly [3].

In this context, over the last years, researches in the field of Ambient Assisted Living (AAL) aim to develop technological solutions which are devoted to enhance the quality of life of elderly population, promoting their independent living, supporting their daily activity and encouraging preventive actions [4].

In a not remote future scenario, service robotic platforms will be machines that aims to cooperate with human-beings to help and assist persons of all ages in daily activities at home, in their workplace and in other environments. They will be able to perform a multitude of roles thanks to their capabilities to act and interact physically, emotionally, socially and safely with humans, providing for an easier and healthier life.

As state from literature evidence, Robot and Information and Communication Technology (ICT) have the potential to enable older adults promoting their independent living and active aging [5]. Personal service robot could be classified into seven main areas according to their main applications: Social interaction, Information, Safety, Health, Leisure, Physical Support and Mobility [6]. For instance, Paro and Nao are developed mainly for leisure purpose [7]. Other robots, like Dustcart, are designed for shopping and garbage transportation [8]. On the contrary, other robots are general purpose, in other words, they are not designed to perform specific tasks, but they could provide a range of services, such as DoRo [9], Care-o-Bot@3 [10] , Pepper [11]. Recently, some of these robots become commercially available, like Buddy from Blue Frog robotics [12], Roomba robot [13] devoted to cleaning task, or Pepper from SoftBank [14] used as a commercial promoter in Japan mall.

In this context, over the last years, several EU projects have been founded to design, develop and test robotic solutions that could support elderly people and stakeholders in daily life tasks.

Within these projects, different robotic platform are customized and tested with real older users to estimate the usability and the acceptability level of the proposed services [15][16][ 17]. For instance, Teresa and Giraff+ projects use Giraff robots; Robot-Era and Astromobile projects customize SCITOS G5 with a co-design approach with end-users. Whereas Kompaii robot is used in Domeo, Mobiserv and Mario projects. It is worth to mention that each of this robot can provide different services aligned with the different projects' aims.

Particularly a total of 13 European service robotics project had been founded between 2011 and 2015 under FP7 and H2020 funding scheme. They are: Mobiserv, SRS, Ksera, Florence, Giraf+, Hobbit, Accompany, Robot-Era, Radio, Mario, Enrich-me, GrowMeUp and Ramcip. The aims of these project are various but could 
be grouped into six big domains namely: Support to the caregiver, Promote Health, Promote social inclusion, promote well-being, physical support, and safety at home. Of course a single project can belong to multiple domains.

If we imagine to cluster the selected projects according to this domains we can obtain an overview of the main service domains investigated by the most relevant service robotic projects. The results of this analysis are reported in Fig. 1.

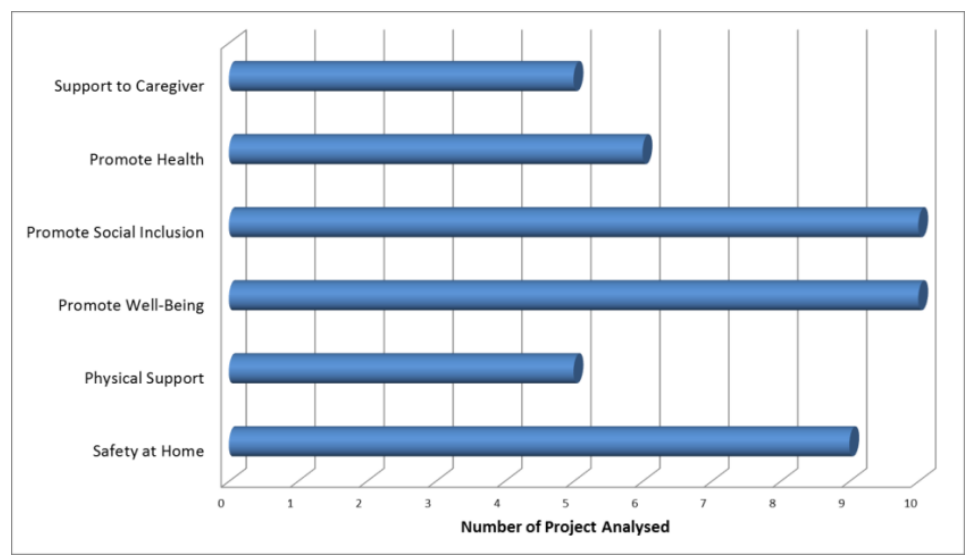

Fig. 1. Service domain distribution

As summarized by this analysis, the past EU projects are mainly devote to the promotion of social inclusions and the well-being. Whereas only 5 projects are focused on the physical support service. Additionally, there is not always a clear reference to the needs of formal and informal caregivers (5 out of 10). Indeed, ICT and robotics solutions often are developed without deeply knowing the end-users' needs and what are the social and infrastructural conditions in which such technology should work. The robotic systems are not extensively tested in real operative conditions. Furthermore, all those projects do not adopt a common methodology to evaluate the effectiveness, the acceptance and the sustainability of the proposed solutions.

In this context, the objective of ACCRA project (Agile Co-Creation for Robots and Aging) is to become the reference co-creation methodology for the development of robotics solutions for aging and to become the reference assessment framework for the evaluation of robotics solutions for aging. ACCRA solutions will be designed and developed to be tested in three different applications: walking support, housework, conversation in four countries (i.e. Italy, France, Netherlands and Japan). For this reason, specific technology has been created and integrated at application, API and platform levels as described in Sect. 5.

Finally, in order to achieve the proposed goals, ACCRA researchers will focus their work on the following aspects: to i) define a methodology for robotics solution for aging based on agile co-creation; ii) develop an assessment framework for impact of robotics solutions for aging; iii) built a platform for agile co-creation 
based on open solutions provided by existing initiatives, integrating important enablers; iv) develop a robotics application for aging focusing on walking support; housework and socialization services.

This paper is organized as follow: Sect. 2 will introduce the ACCRA project, Sect. 3 will present the three applications, Sect 4. details the ACCRA methodology; Sect. 5 and Sect. 6 describes the architecture and the impact of ACCRA solution respectively, finally Sect. 7 concludes the work.

\section{ACCRA project overview}

ACCRA project started in December 2016. It has been founded under H2020 PM14-2016 call as a joint research project between Europe and Japan. The multicultural consortium guarantees complementary expertise from different fields such as engineering, robotic, informatics, social, marketing.

As regard the European partners the researches come from France, Italy and Netherlands. Particularly Trialog, the ACCRA leader, Paris Dauphine University and Bluefrog Robotics come from France; Scuola Superiore Sant'Anna and IRCCS "Casa Sollievo della Sofferenza" come from Italy, and Erasmus University comes from Netherlands. As concern the Japanese side The Kyoto University, ConnectDot and Kobe University belong to ACCRA project.

In order to achieve the proposed goals, the state of art of methodology for design and the technology evaluation in the context of "aging-well" is united to build the ACCRA Methodology. It brings together expertise from robotics, software development, marketing, health services research, and health economics.

\section{ACCRA Applications}

Firstly, it is important to notice that (as remarked in [4] [3]) the understanding of stakeholders' needs play an essential role in the design of acceptable, usable and near-to-market research products. The needs of older citizens are mainly related to the physiological and physical disorder due to natural decline, chronic diseases of physical impairments. Firstly, older persons want to actively contribute to their families and voluntary organization as long as possible, they do not want to be considered as a burden for the family and the society. Additionally, older persons want to reduce negative feelings, like vulnerability and insecurity, and to reduce loneliness and depressions. Furthermore, they want to increase their social activities, because of the decreasing of traditional social network and infrastructure they reduce their social contacts and engagements. Sometimes they need help in managing chronic diseases because complex care procedures can generate confusion. On the basis of these outcomes, ACCRA will develop three robotic applications:

A1) mobility application for aging focusing on walking support, 
A2) daily life application for aging focusing on housework, and

A3) socialization application for aging focusing on conversation.

These application aim at addressing the main elderly needs as depicted in Tab. 1:

Table 1 .Correlation between elderly and caregiver needs [4] and ACCRA applications

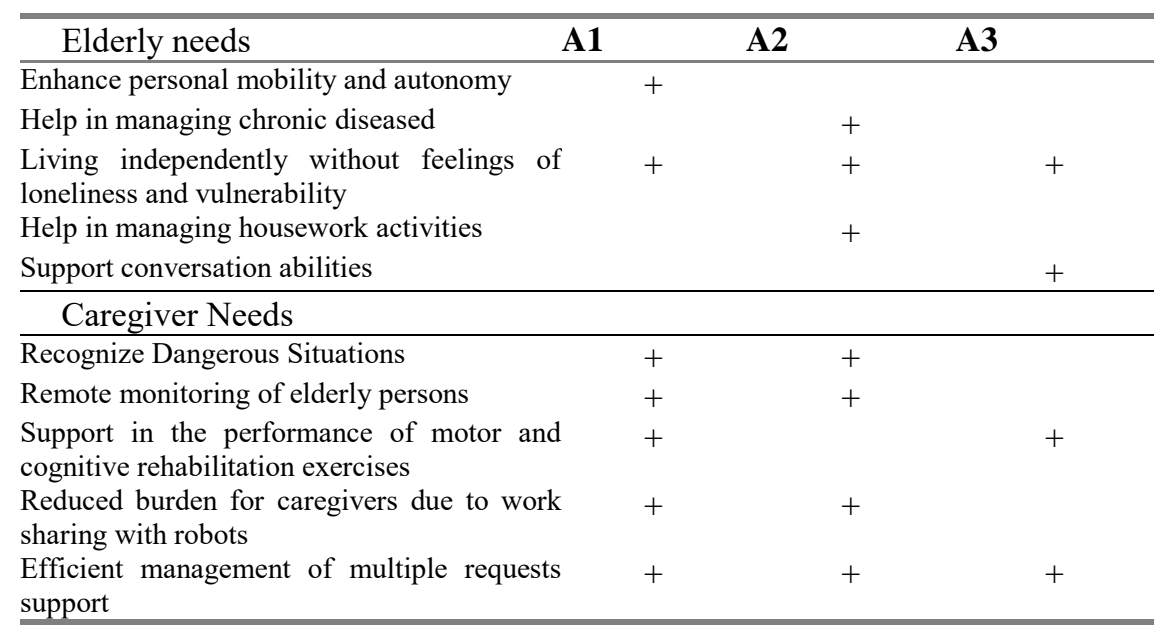

Mobility application will be addressed to people presenting risk of falls or returning from hospitals further to falls. The application will integrate features for physical support for walking (i.e. the robot can physically help). Particularly, the primary goal of this application will be related to the design and the development of the following of robotics features: detection of lack of movement, mobility coaching, help in maintaining independent mobility (Fig. 2).

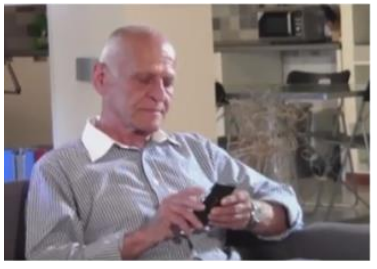

(a)

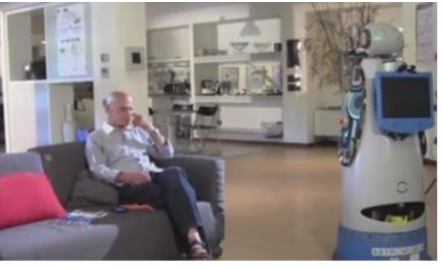

(b)

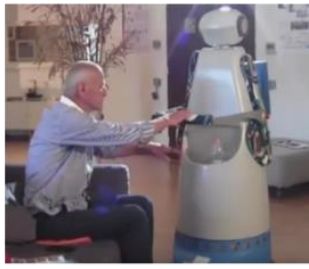

(c)

Fig. 2. Mobility application (a) the elderly user calls ASTRO robot because he needs to go to another room, (b) ASTRO robot arrives (c) the user uses ASTRO handle and together go to the desired room.

Daily life application will be addressed to people with first signs of loss of autonomy (pre-dependency) promoting behaviors favorable for aging well (i.e. mobility, good hydration, social links, etc.) and for detecting signs/risks 
to reduce autonomy. In this context, ACCRA will focus on the demonstration of well-being features through the help of robotics solutions such as the medicine reminder, social links, alerts and diagnosis management.

Conversation rehabilitation. The proposed paradigm will be similar to physical rehabilitation where patients are provided with challenging exercises to stimulate their body; indeed, in ACCRA application, people will be provided with challenging interactions exercises to stimulate their intellectual curiosity on different topics (i.e. fashion and golf) which are modulate on their preferences and psychological profile [18].

Additionally, in order to investigate how the cultural background could influence the personal attitude toward the robotic service. Each application will be refined and tested in different countries as reported in Tab. 2.

Table 2. ACCRA experimentation site

\begin{tabular}{ccccc}
\hline Application & Italy & France & Netherlands & Japan \\
\hline A1 & $\mathrm{x}$ & & $\mathrm{x}$ & \\
$\mathbf{A 2}$ & $\mathrm{x}$ & $\mathrm{x}$ & $\mathrm{x}$ & $\mathrm{x}$ \\
$\mathbf{A 3}$ & $\mathrm{x}$ & & & \\
\hline
\end{tabular}

\section{ACCRA Methodology}

ACCRA proposed methodology distinguishes four steps (see Fig. 3), aligned with the general structure of the ACCRA project:

- Step I: Needs analysis, aim at identifying needs and at investigating the context in which the applications will be used

- Step II: Agile co-creation, aims at developing the robotics solutions in close collaboration with end users, informal and formal caregivers, using agile programming tools.

- Step III: Experimentation, aims at testing the robotics solutions in a real context by a larger group of end users.

- Step IV: Sustainability analysis, aims at defining the potential market for the robotics solutions, and assessing the large scale impact of up scaling robotics solutions on the health system. Particularly, it aims at investigate this scientific and economic aspects: (i) What are the differences between pilots experimenting with the same robot, and what does that say about (cultural) contextual factors? (ii) Is there a potential market for the robot? (iii) What could be the future effects of robots when used more intensively in care organisations and houses? 


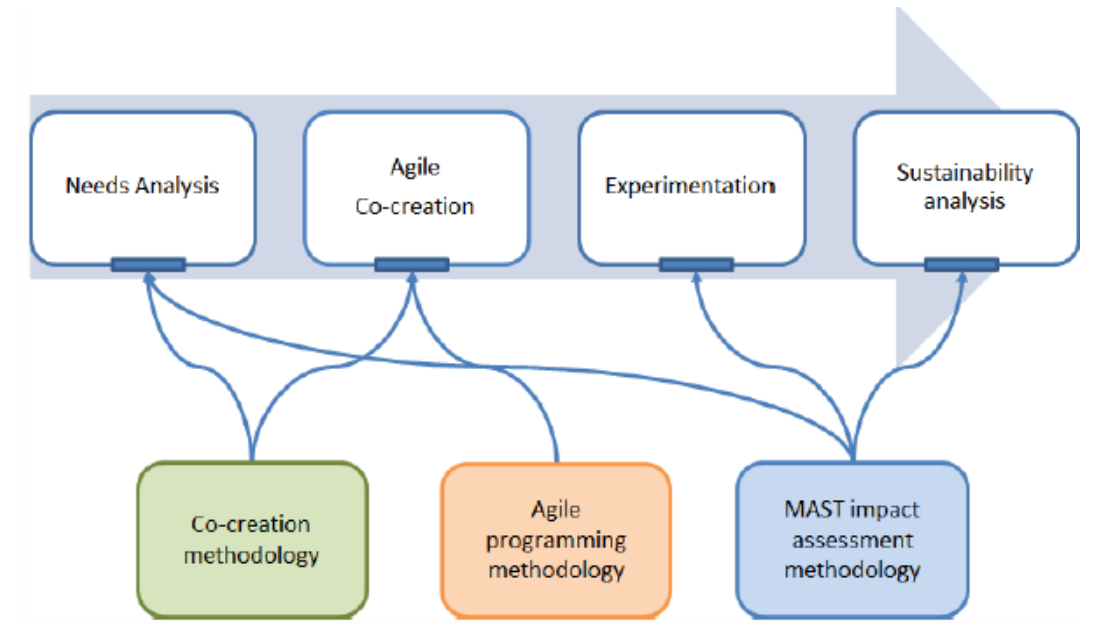

Fig. 3. ACCRA 4-step methodology

The following paragraphs will details step I, step II and step III.

\section{Step I: Needs Analysis}

The purpose of this step is the identification of needs and behaviors of the elderly and the caregivers and, consequently, the re-definition of the applications. The needs analysis is based on a qualitative approach consisting of in-depth interviews. Particularly, it includes twenty in-depth interviews (ten elderly persons and ten formal/informal caregivers) per application and per country.

The interview will be divided into two parts, the first one aims at investigating the general impression of a robot solutions in each particular application. Whereas at the beginning of the second part, ACCRA application will be introduced by using video and introducing possible scenario in order to collect feedback thus to refine the applications. Example of ACCRA scenarios is:

\section{Help to maintain independent mobility}

Stefanie is a 76 years old with difficulties in walking and standing. She is still able to move from a room to another one, but she feels unsafe and needs a constant help. Andrew is a caregiver working in the care facility, that help elderly people to walk from the bed to the restaurant or the gym, but he is not able to accomplish all the requests on-time. For moving from an ambient from another one, Stefanie can use ASTRO robot to get assistance, avoiding Andrew of heavy physical tasks. The service provided by ASTRO can be requested both using a web in- 
terface or interacting directly with ASTRO if in the same room. Stefanie needs to go to the common area to play cards with her friends, she calls ASTRO and together go to the desired place.

\section{Support in housework activities}

Mario is 82 years old and he lives alone in his house since his wife died two years ago. Three years ago he started to suffer from cognitive problems, he has trouble in remember things. Mario needs to be reminded some daily activities like take the correct medicaments at the right moment, eat correctly and follow a regular number of meals per day, preparing the meals correctly avoiding medical restrictions because of high levels of cholesterol. Mario has a help assistant caregiver three times a week to do the household (cleaning, laundry and helping if necessary in bathing). Mario also receives the visit of his/her daughter almost every weekend or week, they are usually in contact. Thanks to ACCRA system the caregiver and the daughter could be always in touch with Mario and receive a feedback on the correct assumption of meals and medicine.

The inclusion/exclusion criteria for mobility, housework, and conversation interview guides are shown below:

1. Mobility

a. Inclusion criteria: 1 ) age $\geq 60$ years; 2 ) elderly with mobility issues (elderly with reduced mobility capabilities, elderly who are at risk of falling, and elderly who are rehabilitating and are in need of a mobility coach); and 3) elderly who gave their informed and signed consent.

b. Exclusion criteria: 1) elderly who do not have any mobility issues (EMS score $>13$ ); and 2) elderly with any cognitive issues.

2. Housework

a. Inclusion criteria: 1) age $\geq 60$ years; 2) elderly who have difficulty engaging in housework; 3) AGGIR grid with the following scores: GIR 4, GIR 5 and GIR 6.

b. Exclusion criteria: 1) elderly who do not have any engaging in housework.

3. Conversation

a. Inclusion criteria: 1) age $\geq 60$ years; 2) Elderly without or with mild cognitive impairment: Mini Mental State Examination (MMSE) score $\geq 24 / 30$; and 3 ) elderly who gave their informed and signed consent.

b. Exclusion criteria: 1) elderly with any cognitive issues: Mini Mental State Examination (MMSE) score $<24 / 30$.

This step of the methodology also includes a thorough analysis of the current situation in each pilot site from a local, regional or even national level. It is important to know about the rules and regulations in each country that are relevant for AAL and robotics, the way the health system is organized (who pays for innovations like these), and the way society perceives technology 


\section{Step II: Agile co-creation}

The central core of ACCRA methodology is the "AGILE co-creation". AGILE cocreation is a developmental approach by which intensive cooperation between users and IT-professionals is the centre [19]. Value co-creation is a collective process through which the interactions of a firm and its customers facilitate the creation of value [20] [21].

As state from literature evidences, researches have not only recognized that value co-creation is a way to achieve service innovation [22] [23], but have also acknowledged the need to further explicate the service innovation structures that facilitate value co-creation [22].

In this context, Information Technologies (IT) play an important role in value cocreation. Technology can enhance the experience environment by enabling a value co-creation platform in which the firm and the customer interact and co-create value together [24].

The important role of the processes used to create IT-enabled products and services that are aligned with the evolving needs of the customer, has received much attention in the literature. Specifically, agile development methods that have gained immense popularity in recent years recognize the central role of customers in the development of products and services [25]. Agile methods prioritize customer satisfaction through the early and continuous delivery of valuable IT solutions, mainly software [26]. In particular, agile methods facilitate fast, intense, focused, and adaptive software development [27-29]. Researchers [24] define agility as "the ability to act fast... and respond quickly to changes."

Agile methods have been recognized for their emphasis on value creation and their potential to facilitate value co-creation by involving customers throughout the project life cycle [30]. In order for managers to successfully co-create value, they need to have the capacity for agility [24]. Recent research and practice has recognized the need to incorporate distributed development through agile practices. However, though agile distributed methods have been recognized for their emphasis on value creation, there is limited research on this topic [31].

On the basis of the aforesaid researches, the main objective of ACCRA project is developing the robotics solutions in close collaboration with end users, informal and formal caregivers, using agile programming tools.

Agile Co-creation of ACCRA robotics solution and service platform is divided in two step:

\section{Sub-step: Services co-creation}

By placing users (i.e. elderly people with loss of autonomy and caregivers) in the centre of the innovation process, the aim of this step in ACCRA is to design a robotic solution and services offering that effectively meet needs, expectations and 
uses of elderly people with loss of autonomy and caregivers. The aim is to improve the robotic solution and services by proposing concrete optimization solutions, perceived as operational both by the elderly people with loss of autonomy, family/professional caregivers and technology/robotics professionals. This approach is strategic in the context of a social innovation project as the ACCRA project. Indeed, researchers in social innovation [32] show that the diversity of actors involved in the innovation process and the active participation of users (here, the elderly with loss of autonomy and family and professional caregivers) [33]. Features are essential in the creation and implementation of new solutions. This provides a more complete representation of the problems, causes and possible solutions and allows to better respond to the problems of individuals by developing better solutions. This finally encourages the implementation of the identified solutions [33]. From the first prototypes developed by the consortium, a working group (co-creation group) is created per application and per pilot site, consisting of seniors losing their autonomy (corresponding to use cases), informal (e.g. family) or formal caregivers (e.g. nurse, physiotherapist), technology/robotics professionals, and researchers with a double expertise on elderly people and marketing services. These co-creation groups will be working on the optimization of ACCRA robotic solutions and services platform to best meet the users' needs. Primarily these are the needs of the elderly people with loss of autonomy, but also family and professional caregiver needs are incorporated, because the use of robotics is also meant to complement or even substitute their tasks. The process embroils elderly people with loss of autonomy and family/professional caregivers who test the robot and the related service platform (on the basis of a prototype), a first co-creation group that envolves elderly with loss of autonomy, informal caregivers and professional caregivers who are asked to deliver constructive criticism on previously experienced services (explaining how ACCRA services, functionality and ergonomics of the robotic support meet their needs and uses). The multidisciplinary group then exchange on possible improvement tracks and selects the priority axes of rework. After the first group, the ACCRA consortium reworks the robot and related service platform, and the optimized version of the robotic solution will be again submitted to the co-creation group. At least three other iterations of the group then take place, according to the previously exposed process

\section{Sub-step: Agile Programming}

Fig. 4 explains the principles of conventional agile programming: product development is carried out in short periods called sprints. The objectives of sprints are agreed by the agile programming team (e.g. engineers who apply the agile approach), the product manager and the product owner. The result of a sprint is executable and can be demonstrated. It can be therefore assessed by both the product owners and managers. Because of this constraint, there is pending work that is not mature yet to be integrated. It is managed through project and iteration backlogs. 
There are a wide number of methodologies and framework available such as SCRUM [34].

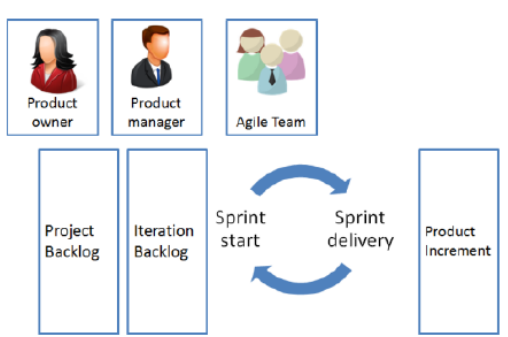

Fig.4. Conventional Agile Programming

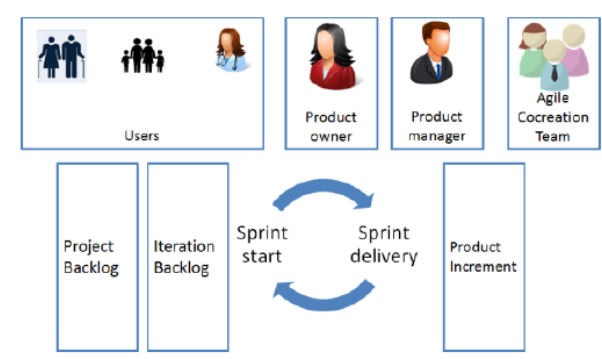

Fig.5. ACCRA Agile Programming

Fig. 5 shows how agile programming will be adapted to ACCRA. The agile team is now the agile co-creation team, i.e. it includes specialists of co-creation. As co-creation is user centric, users (elderly, family, carers) are also part of the overall team.

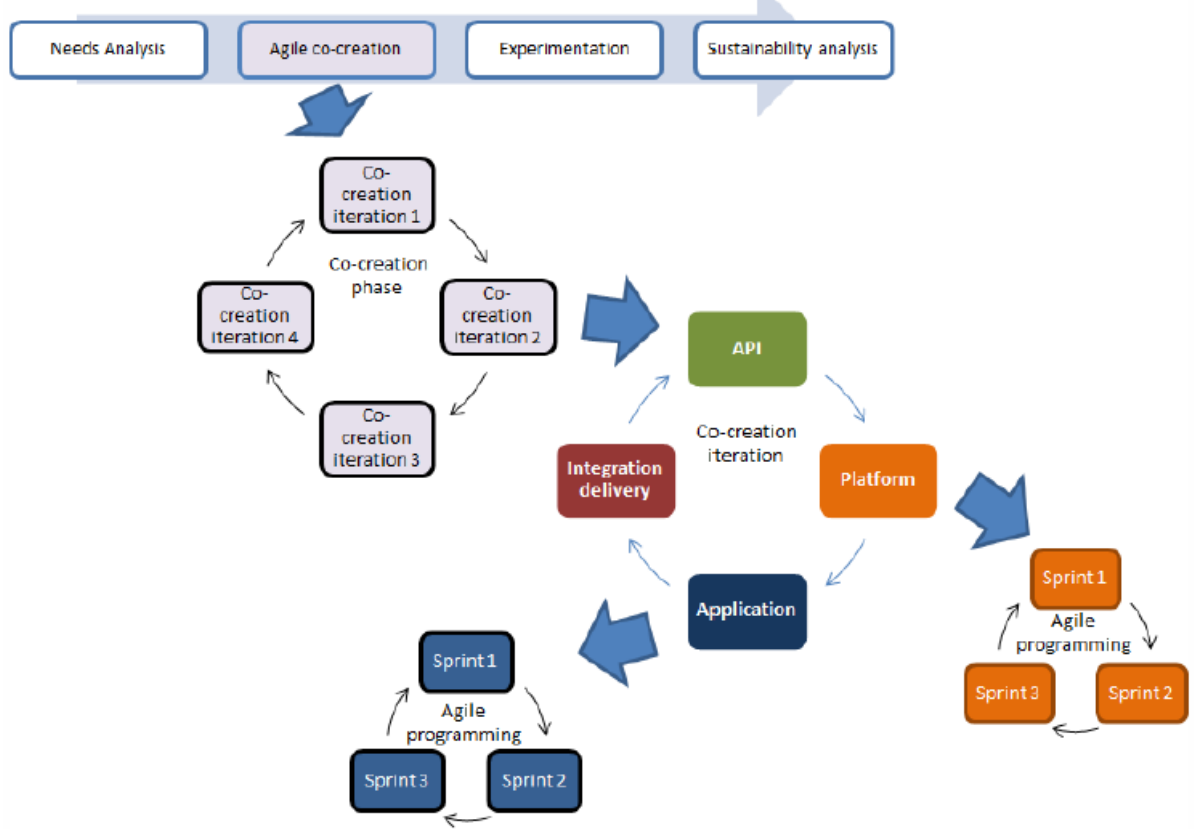

Fig. 6. Integration of Co-Creation with Agile Programming.

It is important however in the ACCRA methodology to ensure that the members of the agile cocreation team as well as their interactions with users are at the right level. This will be one of the important challenges of the project. The proposed approach is depicted in Fig. 6: 
1. It starts with the ACCRA methodology 4 steps

2. The co-creation phase is iterative

3. Each co-creation phase consists of three programming steps:

a. Application modifications/extensions

b. Resulting API modifications/extensions

c. Resulting Platform modifications/extensions

4. Applications modifications/extensions are then subject to a number of sprints (at least 1)

5. Platform modifications/extensions are also subject to a number of sprints (at least 1)

6. As expected, application sprints and platform sprints are carried out separately in parallel. For integration reasons, a common final sprint could be organized.

\section{Step III: Experimentation}

The co-creation is followed by an experimentation in France, Italy, Netherlands and Japan (Tab. 2). Both in this step and in step 4 the MAST model (Model for Assessment of Telemedicine) will be used as a guideline, although it will be adapted to the robotics context where needed [35].

The MAST model is an international, validated model, which was originally developed for telemedicine, but has been applied in other domains as well. It is used for the assessment of costs and effects of innovations from a multidimensional perspective (also taking contextual factors such as organizational, ethical and legal issues into account). MAST has been used in large telemedicine studies like RCTs, but it is also relevant for smaller experimentations, such as the one done in ACCRA. One of the strong points is that it also involves the outcome of all the actors involved.

The use of the MAST evaluation framework facilitates the assessment of effectiveness and contribution to the quality of care of the robotics application. It includes three elements: (1) Preceding considerations, (2) Multidisciplinary assessment and (3) Transferability assessment. The experimentation phase of ACCRA is based on the second element of MAST: multidisciplinary assessment.

\section{ACCRA architecture}

From the point of view of system architecture, the main important feature of the ACCRA architecture is the use of a system integration approach between robotics devices and intelligent living environments, which can support novel service de- 
livery models, including the integration of robots, sensor networks, and handling data in the cloud, in a cloud robotic approach.

Recently, Cloud Robotics has been defined as "Any robot or automation system that relies on either data or code from a network to support its operation, i.e., where not all sensing, computation, and memory is integrated into a single standalone system [36]."

According to this specific, ACCRA solution is based on the FIWARE cloud platform. The FIWARE platform is characterized by a simple set of APIs (Application Programming Interface) the ease the use of the platform and the development of Smart Applications. Besides, FIWARE provided a multitude of FIWARE components (also referred as enablers) that can be easily combined to perform more complex tasks. FIWARE enablers cover a wealth of applications in several domains, including IoT, Big data and Cloud Computing. Fig. 7 shows how ACCRA technology will be constructed: applications uses APIs (application programming interfaces) that allow access to the platform capabilities. The capabilities are structured through simpler enablers. Fig. 7 also shows that applications, APIs and platform capabilities can be located at different levels, i.e. cloud and network level, device and IoT level. For instance a robotics solution could run entirely at the robot level, without any issues on networks or cloud accesses . It can as well run as a distributed solution, with the application running at network level and making use of platform capabilities running at network and robot level.

According to specific addresses of ACCRA project, three types of applications are involved as described in the previous section.

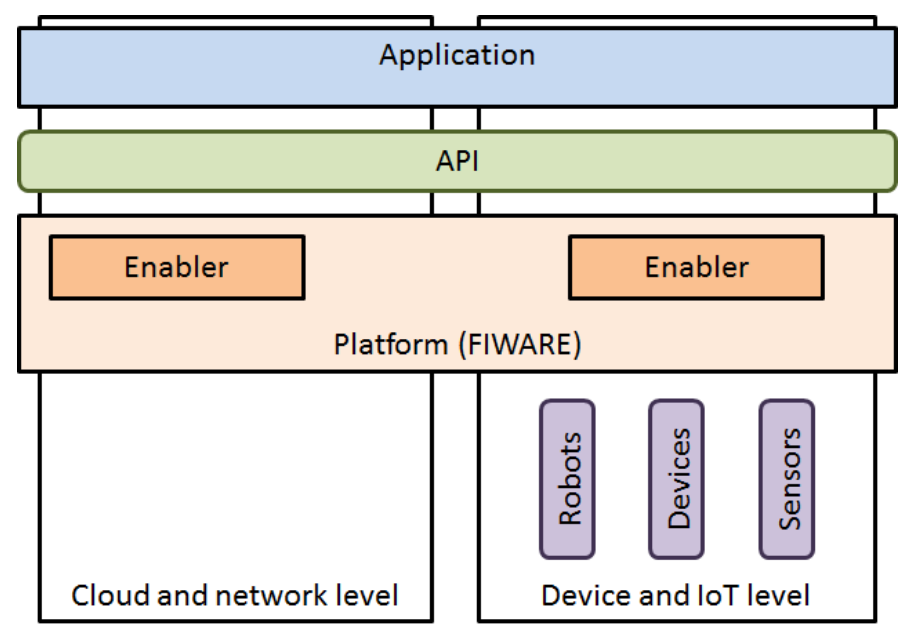

Fig. 7. ACCRA system architecture concepts 
As previously mentioned, the whole platform is based on FIWARE, for integration of capabilities already available, as management of personal data through the security enablers or processing of complex event through data management enabler.

Furthermore, in order to demonstrate the generalization performed during ACCRA project, two different robotic platforms are involved in the project: Astro (for Application 1 - Support and coach for walking) and Buddy (for application 2 housework, and application 3 - conversation).

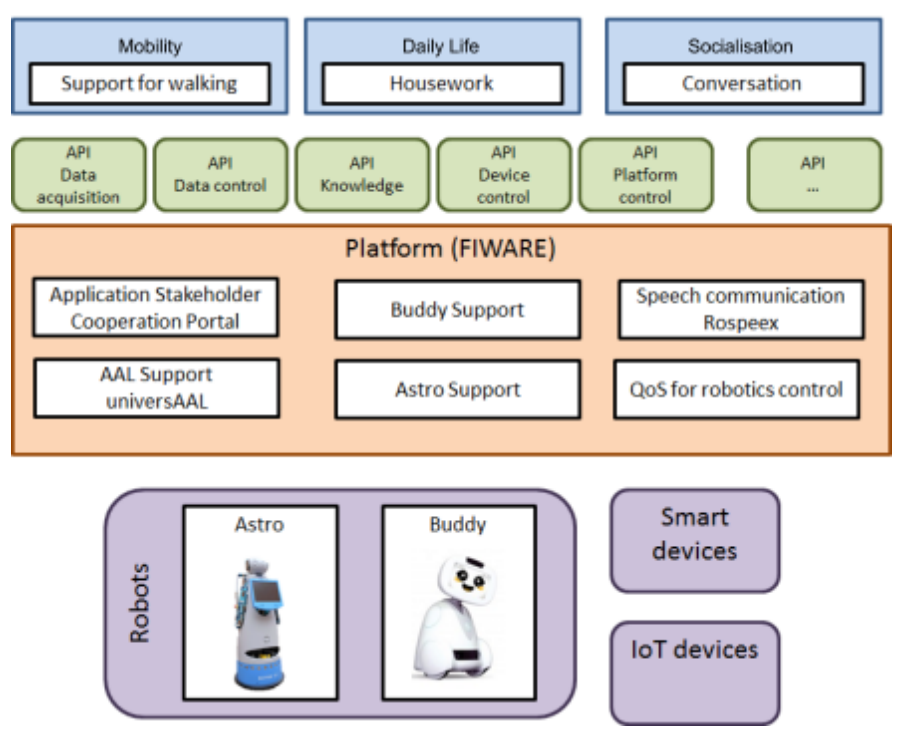

Fig. 8. ACCRA system architecture components

Astro is an assistive smart robotic platform dedicated to mobility and user interaction. It has been designed for moving within unstructured home and residence environments. It is a big robot, solid enough to become a smart walker. It can identify the location of the user at home and to interact with him using natural language, touch screen and visual LED system. On its back, the robot has an adaptable physical support to help people to stand up. Along the ACCRA project, the work on Astro robot is aiming at improving its smart walker capability and other services.

Buddy robot is the physically opposite of Astro robot. It is a small-size robot and designed as a companion home. He cannot be physically a support for walking. The Development Tools (SDK), based on the Open-Source technologies such as Unity3D (video games) and OpenCV (aimed at real-time computer vision) to develop advanced applications for the robot. Along the ACCRA project, the work on Buddy is aiming at integrating new applications and potentially new hardware in 
order to meet the use cases requirements in a different approach than the Astro robot. The Buddy robot can be connected to the home and internet environment in order to fulfil its tasks. In Fig. 8, the ACCRA architecture is summed up.

\section{Discussion}

The greatest challenge for Europe and Japan is to create a sustainable health system. In this context, AAL researches focus they efforts onto three main pillars: Prevention, Compensation and Support and Independent Living [4]. These pillars represent the effect that AAL services have on the life of older persons. Prevention pillar focuses mainly on action to avoid or delay the onset of morbidity. Compensation and Support pillar includes the AAL services and devices that compensate physical and cognitive limitations. Whereas Independent Living pillar includes all the services and activities that allow older people to live independently and to participate to the community life. These pillars are complementary and partially overlapped because the same service can produce effects in different areas. ACCRA will develop and demonstrate three applications for two out of three pillars, particularly for "Compensation and Support" and "Independent living", since the three scenarios are mainly focused on these aspects:

- Supporting indoor mobility

- Problems with ADL tasks and housework

- Lack of social interaction

Additionally, in order to overcome the limitation of the previous research works [6], the proposed applications are co-created with users to optimally meet their needs. Furthermore, in the extensive experimentation phase the impact of the robots on daily life will be studied using a multidisciplinary assessment.

Additionally, ACCRA services will not be evaluated in a lab environment, but in real care organisations and private homes of four different countries (i.e. France, Italy, Netherlands and Japan). This approach allows ACCRA researches to investigate how the cultural differences and attitudes could influence the perceived usefulness toward robotic solutions. It is important to notice that, because of the relatively small sample size per application and per pilot, a complementary research is planned through a scenario analysis to corroborate the statistical significance of the results. This action, indeed, will improve the robustness of the results, so that impacts on quality of life in the broadest sense (covering autonomy, health, wellbeing, safety, dignity, etc.) can be demonstrated.

If elderly people can live independently as long as possible, this will have a financial impact on the health system but also have an impact on the roles in health care: older persons and their carers will be more independent and autonomous [37]. In this sense, the results of ACCRA project can be used to forecast health system sustainability. The number of users, and duration of experimentation is rel- 
atively small, but the data collection gives a basic understanding of the types of advantages robotics will have for the health systems.

It is worth to mention that ACCRA project will focus they efforts on the definition of carers and family needs in order to include in the co-creation process also these points of view which could enrich the design and the evaluation phases.

Summarizing ACCRA will have an impact on four aspects:

- Agile co-creation: ACCRA project aims to constitute of a FIWARE-based platform environment for agile co-creation of robotics solutions for aging, focusing on the needs of the end users (elderly persons and formal/informal caregivers), integrating domain specific capabilities.

- Open solutions: ACCRA project relies on technologies and enablers which are mostly open platforms. Indeed, FIWARE, universAAL, Rospeex are all open source solutions that will be used to build the ACCRA platform. ACCRA researches will use existing standards, supported by the European Community, thus, thanks to the international consortium, ACCRA project contributes to further adoption of open platforms internationally.

- Interoperability: ACCRA co-creation approach also integrates flexible APIs definitions. It therefore promotes a practice that will allow for adoption and therefore agreement on interoperability aspects. ACCRA methodology promotes "interoperability-by-design", i.e. interoperability requirements are integrated in the creation process.

- Methodology: As ACCRA focuses on a novel co-creation methodology (1) that fully integrates programming and (2) that is dedicated for the development of robotics solutions, ACCRA plans to seek standardisation of its agile co-creation methodology. Different standardisation options are possible, e.g. focusing on ergonomics related standards, on system engineering related standards, on robotics related standards, or on health informatics related standards.

\section{Conclusion}

In this paper, an overview of ACCRA project has been presented. Within ACCRA project an integrated framework for cloud robotics-based solutions will be developed and experimented to promote active and healthy aging. The proposed methodology integrates both co-creation and agile programming. It includes all the elements for flexibility, for user/stakeholder centric design and for multidimensional evaluation. It has been conceived to overcome the limitation of the other robotics projects and to became a referring methodology for this kind of project. ACCRA architecture will be developed on open platforms such as ROS, FIWARE, Rospex and universALL in order to promote the adoption of this platform in the scientific community. Additionally, ACCRA focus its effort on the analysis of cultural and economic aspects on ACCRA's applications in four countries (Italy, France, Netherlands and Japan). 


\section{Acknowledgments}

This work was supported by the ACCRA Project, founded by the European Community's Horizon 2020 Programme (H2020-SCI-PM14-2016) - grant agreement No. 738251

\section{Reference}

1. Population aging in Europe, European Commission available at: https://ec.europa.eu/research/social.../pdf/.../kina26426enc.pdf (Accessed: May 2017).

2. Innovation for Age-Friendly Buildings, Cities and Environments available at: http://ec.europa.eu/research/innovation-union/pdf/active-healthyaging/d4 achievements 2015.pdf (Accessed: May 2017).

3. van den Broek, G., Cavallo, F., \& Wehrmann, C. (2010). AALIANCE ambient assisted living roadmap (Vol. 6). IOS press.

4. Moschetti, A., Fiorini, L., Aquilano, M., Cavallo, F., \& Dario, P. (2014). Preliminary findings of the AALIANCE2 ambient assisted living roadmap. In Ambient assisted living (pp. 335-342). Springer International Publishing.

5. Czaja, S. J. (2016). Long-term care services and support systems for older adults: The role of technology. American Psychologist, 71(4), 294.

6. Esposito, R., Fiorini, L., Limosani, R., Bonaccorsi, M., Manzi, A., Cavallo, F., \& Dario, P. (2015). Supporting active and healthy aging with advanced robotics integrated in smart environment. Optimizing assistive technologies for aging populations, 46-77.

7. Suzuki, R., \& Lee, J. (2016, November). Robot-play therapy for improving prosocial behaviours in children with Autism Spectrum Disorders. In Micro-NanoMechatronics and Human Science (MHS), 2016 International Symposium on (pp. 1-5). IEEE.

8. Ferri, G., Mondini, A., Manzi, A., Mazzolai, B., Laschi, C., Mattoli, V., ... \& Dario, P. (2010, May). DustCart, a mobile robot for urban environments: Experiments of pollution monitoring and mapping during autonomous navigation in urban scenarios. In Proceedings of the IEEE International Conference on Robotics and Automation (ICRA 2010) Workshop on Networked and Mobile Robot Olfaction in Natural, Dynamic Environments.

9. Bevilacqua, R., Felici, E., Marcellini, F., Glende, S., Klemcke, S., Conrad, I., ... \& Dario, P. ,(2015, August). Robot-era project: preliminary results on the system usability. In International Conference of Design, User Experience, and Usability (pp. 553-561). Springer International Publishing.

10. Reiser, U., Jacobs, T., Arbeiter, G., Parlitz, C., \& Dautenhahn, K. (2013). Care-O-bot ${ }^{\circledR} 3-$ Vision of a robot butler. In Your virtual butler (pp. 97-116). Springer Berlin Heidelberg.

11. Aaltonen, I., Arvola, A., Heikkilä, P., \& Lammi, H. (2017, March). Hello Pepper, May I Tickle You?: Children's and Adults' Responses to an Entertainment Robot at a Shopping Mall. In Proceedings of the Companion of the 2017 ACM/IEEE International Conference on Human-Robot Interaction (pp. 53-54). ACM.

12. Bluefrog robotics official website: http://www.bluefrogrobotics.com/en/home/ (accessed: May 2017).

13. Roomba robot official website: https://www.roomba.it/ (Accessed: May 2017)

14. Softbank robotics, Peper robot sales official website: https://www.ald.softbankrobotics.com/en/Launch Sales of Pepper (Accessed: May 2017).

15. Fiorini, L., Esposito, R., Bonaccorsi, M., Petrazzuolo, C., Saponara, F., Giannantonio, R., .. \& Cavallo, F. (2016). Enabling personalised medical support for chronic disease management through a hybrid robot-cloud approach. Autonomous Robots, 1-14. 
16. Sancarlo, D., D’Onofrio, G., Oscar, J., Ricciardi, F., Casey, D., Murphy, K., ... \& Greco, A. (2016, June). MARIO Project: A Multicenter Survey About Companion Robot Acceptability in Caregivers of Patients with Dementia. In Italian Forum of Ambient Assisted Living (pp. 311-336). Springer, Cham.

17. Khosla, R., Nguyen, K., \& Chu, M. T. (2017). Human Robot Engagement and Acceptability in Residential Aged Care. International Journal of Human-Computer Interaction, 1-13.

18. Sugiura, K., \& Zettsu, K. (2015, September). Rospeex: A cloud robotics platform for humanrobot spoken dialogues. In Intelligent Robots and Systems (IROS), 2015 IEEE/RSJ International Conference on (pp. 6155-6160). IEEE.

19. User requirements \& Co-creation: an Agile approach. Posted May 30, 2014. https://legoviews.com/2014/05/30/user-requirement-agile-cocreation/. Accessed May 4, 2017.

20. Grönroos, Christian, \& Voima, Päivi. (2013). Critical service logic: making sense of value creation and co-creation. Journal of the Academy of Marketing Science, 41(2), 133-150.

21. Ramesh, Balasubramaniam, Mohan, Kannan, \& Cao, Lan. (2012). Ambidexterity in Agile Distributed Development: An Empirical Investigation. Information Systems Research, 23(2), 323-339.

22. Lusch, Robert F., \& Nambisan, Satish. (2015). Service Innovation: A Service-Dominant Logic Perspective. MIS Quarterly, 39(1), 155-176.

23. Vargo, Stephen L, Maglio, Paul P, \& Akaka, Melissa Archpru. (2008). On value and value cocreation: A service systems and service logic perspective. European Management Journal, 26(3), 145-152.

24. Prahalad, C. K., \& Ramaswamy, Venkat. (2004). The future of competition: Co-Creating Unique Value with Customers. Harvard Business School Press, Boston, MA.

25. Barlow, Jordan B. Keith Mark JeffreyWilson David W. Schuetzler Ryan M. Lowry Paul BenjaminVance AnthonyGiboney Justin Scott. (2011). Overview and Guidance on Agile Development in Large Organizations. Communications of the Association for Information Systems, 29, 25-44.

26. Fowler, Martin, \& Highsmith, Jim. (2001). The agile manifesto. Software Development, 9(8), 28-35.

27. Cao, Lan, Mohan, Kannan, Xu, Peng, \& Ramesh, Balasubramaniam. (2004). How extreme does extreme programming have to be? Adapting XP practices to large-scale projects. Paper presented at the System Sciences, 2004. Proceedings of the 37th Annual Hawaii International Conference on.

28. Cao, Lan, Mohan, Kannan, Xu, Peng, \& Ramesh, Balasubramaniam. (2009). A framework for adapting agile development methodologies. European Journal of Information Systems, 18(4), 332-343.

29. Highsmith, Jim, \& Cockburn, Alistair. (2001). Agile software development: The business of innovation. Computer, 34(9), 120-127.

30. Babb, J, \& Keith, Mark. (2011). Co-creating value in systems development: A shift towards service-dominant logic. AMCIS 2011 Proc-All Submissions. Paper, 456.

31. Madi, Tamer, Dahalin, Zulkhairi , \& Baharom, Fauziah (2013). Towards a user value cocreation model for agile web development approach. Science International, 25(4).

32. Harrisson, D., et Vézina, M. (2006). L'innovation sociale : une introduction. Annals of Public and Cooperative Economics, 77, 2, 129-138.

33. Lallemand, D. et l'ANAS-FNARS (2001), Les défis de l'innovation sociale. Issy-lesMoulineaux : ESF Editeur.

34. SCURM official website: www.scrum.org (Accessed: May 2017).

35. Kristian Kidholm et al.: MAST Manual, February 2010, http://www.telemed.no/methotelemed.4565273-125741.html, p.3.

36. Kehoe, B., Patil, S., Abbeel, P., \& Goldberg, K. (2015). A survey of research on cloud robotics and automation. IEEE Transactions on Automation Science and Engineering, 12(2), 398409. 
37. Turchetti, G., Micera, S., Cavallo, F., Odetti, L., \& Dario, P. (2011). Technology and innovative services. IEEE pulse, 2(2), 27-35. 